\title{
Efficacy of Trimebutine Maleate Combined With Hyperbaric Oxygen Therapy on Irritable Bowel Syndrome
}

\author{
XINLI FENG, YAMEI WANG ${ }^{1}$, YING CHANG ${ }^{2}$, CHUHAI WANG ${ }^{3}, \mathrm{JUAN}^{\mathrm{J} I^{4}}$, QUANWEI DI ${ }^{5}$ AND MENG SUN ${ }^{6 *}$ \\ Department of Hyperbaric Oxygen, 'Department of Infection Control, ${ }^{2}$ Department of Gastroenterology, ${ }^{3}$ Department of \\ Neurology, ${ }^{4}$ Department of Nephrology, ${ }^{5}$ Department of Internal Medicine, Worker's Hospital of China Lucky Group \\ Corporation, ${ }^{6}$ Department of Interventional Vascular Medicine, Affiliated Hospital of Hebei University, Baoding, Hebei 071000, \\ China
}

Feng et al.: Efficacy of Trimebutine Maleate Combined with Hyperbaric Oxygen Therapy

\begin{abstract}
To determine the efficacy of trimebutine maleate combined with hyperbaric oxygen therapy on irritable bowel syndrome. Totally 180 patients diagnosed as irritable bowel syndrome who were admitted to our hospital between June 2016 and June 2019 were enrolled and randomized into the control group and the observation group according to the number of visits (each $n=90$ ). The control group was treated with trimebutine maleate through oral medication, while the observation group was given hyperbaric oxygen therapy additionally based on the treatment for the control group. Both groups were treated for $4 \mathrm{w}$. Then the following items of the two groups before and after intervention were evaluated and compared: serum neurotransmitterassociated parameters (5-hydroxytryptamine, somatostatin, substance $P$, neuropeptide $Y$ ), rectal manometry parameters (rectal sensation threshold, rectal defecation threshold, rectal pain threshold and rectal motor index), self-rating anxiety scale score and efficacy. Before treatment, no notable difference was found between the two groups in rectal manometry indices, serum neurotransmitter-associated indices and self-rating anxiety scale score (all $\mathbf{p}>\mathbf{0 . 0 5}$ ). After treatment, the rectal manometry indices, serum neurotransmitter-associated indices and self-rating anxiety scale score of both groups were greatly improved (all $\mathbf{p}<0.05)$ and all these improvements in the observation group were more significant than those in the control group (all $\mathbf{p}<0.05)$. In addition, the observation group got notably higher efficacy than the control group $(p<0.01)$. Trimebutine maleate combined with hyperbaric oxygen therapy is effective in the therapy of irritable bowel syndrome, without toxic and side effects, so it is worthy of clinical application.
\end{abstract}

Key words: Trimebutine maleate, hyperbaric oxygen, diarrhea, irritable bowel syndrome, hypersensitivity

Irritable Bowel Syndrome (IBS) is a pervasive chronic gastrointestinal functional condition. Patients with it are mainly manifested by gastrointestinal dysfunction accompanied by abnormal defecation, abdominal pain, diarrhea, abdominal distension and distress after defecation and it can be basically classified into constipation type, diarrhea type and mixed type according to its symptoms ${ }^{[1,2]}$. IBS is the result of the interaction of many factors and its pathogenesis is still under investigation. It may be bound up with factors such as abnormal gastrointestinal dynamics, genetics, psychological stress, visceral hypersensitivity, abnormal immunity, intestinal inflammation and infection ${ }^{[3]}$. Patients with it are often accompanied by mental problems such as anxiety and insomnia ${ }^{[4,5]}$, which greatly compromises their physical and mental health and life quality ${ }^{[6]}$. Clinically, IBS is still mainly

*Address for correspondence

E-mail: sunmeng135135@sina.com treated by comprehensive therapy based on drug therapy and no single drug has been verified to be effective for it. This study adopted trimebutine maleate combined with Hyperbaric Oxygen (HBO) based on symptomatic treatment to treat IBS. Totally 180 patients who were admitted to the Affiliated Hospital of Hebei University between June 2016 and June 2019 and met the internationally recognized Rome II diagnostic criteria for IBS were enrolled ${ }^{[7]}$. They were consisted of 82 males and 98 females, at $(44.8 \pm 6.6)$ y old on average (the maximum age: $60 \mathrm{y}$ old; the minimum age: $28 \mathrm{y}$ old), with a mean course of disease of (14.4 \pm 7.5$)$ mo (the shortest course: $4 \mathrm{mo}$; the longest course: $37 \mathrm{mo}$ ). The study was carried out with permission from the Hospital Ethics Committee. The enrolled patients were randomized into the control group and the observation group according to their number of visits (each $n=90$ ). The two groups were not greatly different in general 
data including gender, age and course of disease, type of disease, education level and occupation (all $\mathrm{p}>0.05$, Table 1). The inclusion criteria includes patients confirmed with IBS, patients willing to cooperate with the study after being apprised of the study, patients without comorbid diseases affecting the treatment and research in this study, such as heart disease, liver disease, kidney disease, diabetes, hyperthyroidism, congenital megacolon and anorexia nervosa, patients without intestinal organic diseases after confirmation, patients who used drugs according to regulations and those with detailed case information. The exclusion criteria include patients with contraindications for HBO therapy. Patients in the control group were treated with trimebutine maleate (Shangxi sxante Biopharmaceutical Co., Ltd., specification: $0.1 \mathrm{~g}$; another name: Ruijie). It was orally taken at 2 granules/times, 3 times/d and 30 min before each meal. Patients in the observation group were treated with HBO therapy additionally based on treatment for the control group. For those who received HBO for the first time, staff were arranged to introduce the main treatment mechanism and treatment method and inform them of the difference and different effects between $\mathrm{HBO}$ and atmospheric oxygen inhalation. HBO was carried out with YC2475/0.3-101V air compression chamber for multiple individuals at absolute atmospheric pressure (ATA) of 2.0. Each patient was let to inhale oxygen 30 min twice, with an intermission of $10 \mathrm{~min}$. During treatment, compression and decompression were conducted for $20 \mathrm{~min}$, respectively and the treatment was conducted once a day for 7 consecutive $d$ (a course of treatment). Both groups were treated for $4 \mathrm{w}$ in total and they were followed up once in the $1^{\text {st }}$ mo and once in the $3^{\text {rd }}$ mo after treatment to record their detailed symptom changes and related conditions. The efficacy on the two groups was evaluated after four courses of treatment. The hierarchical counting method was adopted to score the symptoms of the two groups before and after treatment, including abdominal pain, diarrhea, abdominal distension, incomplete defecation, mucus defecation and improvement of stool frequency, change of stool characteristics, insomnia, dreaminess, fatigue, irritability and anxiety. According to the criteria; 0 points were given for no symptoms, 1 point for mild symptoms, 2 points for moderate symptoms and 3 points for severe symptoms. The symptom scores were aggregated. The efficacy was evaluated according to the reduction rate of the total symptom score $(\eta)^{[8]}$. $\eta=$ (aggregated score before treatment-aggregated score after treatment)/the aggregated score before treatment $\times 100 \%$. Cured: The symptoms and signs 149 disappeared or basically disappeared after treatment and $\eta \geq 95 \%$; markedly effective: The symptoms and signs were alleviated obviously after treatment and 70 $\% \leq \eta<95 \%$; Effective: The symptoms and signs were alleviated after treatment and $30 \% \leq \eta<70 \%$; Ineffective: The symptoms and signs were not alleviated and even aggravated after treatment and $\eta<30 \%$. Total effective rate $(\%)=($ Number of cured patients + Number of markedly effectively treated patients + Number of effectively treated patients)/the total number of patients $\times 100 \%$. The contents of 5-hydroxytryptamine (5-HT), Somatostatin (SS), Substance P (SP) and neuropeptide Y (NPY) in the venous blood serum of the two groups were quantified with an Enzyme-Linked Immunosorbent Assay (ELISA) before and after treatment. The rectal motor index, rectal sensation threshold, rectal defecation threshold and rectal pain threshold of the two groups were measured a by PC Polygraf High Resolution (HR) multichannel gastrointestinal function tester (CTD-Synetics Medical Company, Sweden) at admission and within $15 \mathrm{~min}$ after treatment. The Self-Rating Anxiety Scale (SAS) was adopted for evaluating the anxiety of the two groups before treatment and after 4 courses of treatment ${ }^{[9,10]}$, in which a SAS score of 60-69 points indicated moderate anxiety and a SAS score of 69 points indicated severe anxiety. Statistical Package For The Social Sciences (SPSS) 22.0 statistical software was used for data analysis. Enumeration data, expressed as $\%$, were compared between groups using the $\chi^{2}$ and measurement data, expressed as the $(\mathrm{x} \pm \mathrm{s})$ were analyzed by the $t$ test. $p<0.05$ indicates a difference and $p<0.01$ indicates a remarkable difference. In terms of clinical efficacy, the observation group got a notably higher total effective rate than the control group $(\mathrm{p}<0.01$, Table 2 ). Before treatment, the two groups were not greatly different in serum neurotransmitter-associated indices including 5-HT, SS, SP and NPY (all p>0.05), After treatment, serum SP, SS and 5-HT in both groups declined notably (all $\mathrm{p}<0.01$ ), while serum NPY in them increased notably $(\mathrm{p}<0.05)$ and then declines and increase in the observation group were all more notably than those in the control group (all $\mathrm{p}<0.01$ ) (Table 3). Before treatment, no notable difference was found between the two groups in rectal sensation threshold, rectal defecation threshold, rectal pain threshold and rectal motor index (all $\mathrm{p}>0.01$ ), while after it, these indices of the observation group were all superior to those of the control group (all $p<0.01$, Table 4 ). Before treatment, the two groups were not greatly different in SAS score $(\mathrm{p}>0.05)$. After $4 \mathrm{w}$ of treatment, the SAS score of both groups decreased and the score of the maceutical Sciences 
observation group was notably lower than that of the control group $(\mathrm{p}<0.01)$ (Table 5). As a pervasive chronic digestive tract disease, IBS has a global incidence of $1.1 \%-45.0 \%$. In China, the incidence of IBS (Rome II Standard) is over $5 \%$ and is on the rise and its outpatient rate accounts for $30 \%$ to $70 \%$ of the digestive specialist outpatient clinic worldwide ${ }^{[11]}$. Trimebutine maleate, a common drug for regulating gastrointestinal abnormalities, has a bidirectional regulating influence on gastrointestinal smooth muscle ${ }^{[12]}$. When the motility rhythm of the gastrointestinal tract is too low, it can act on adrenal receptors by reducing the synthesis and release of adrenaline and increasing the rhythm. When the motility rhythm of it is hyperactive, it can act on choline receptors by reducing choline release and lower the hyperactivity of digestive tract motility, thus helping balance the digestive tract rhythm. HBO originated in the United States in the 1960s has been widely adopted in the treatment of diseases like gas gangrene, bubble embolism, gas poisoning, chronic osteomyelitis, concussion sequelae, bronchial asthma, acute central retinitis, sudden deafness, diabetic foot and cerebral infarction $^{[13,14]}$. In China, Hongping et al. ${ }^{[15]}$ applied HBO to treat IBS for the first time, but they only mentioned that $\mathrm{HBO}$ could reduce mucosal exudation and mucus secretion and did not further report and elaborate the influence of HBO on various physiological indexes of patients with IBS and its mechanism of action. In addition, there are few research reports on trimebutine maleate combined with $\mathrm{HBO}$ in treating IBS. The present study investigated the efficacy of trimebutine maleate combined with $\mathrm{HBO}$ on IBS, compared and analyzed the related indexes before and after treatment. Our research results and possible causes are as follows: Before treatment, the two groups were not greatly different in rectal manometry indices (rectal sensation threshold, rectal defecation threshold, rectal pain threshold and rectal motor index), blood neurotransmitter-associated indices (5-HT, SS, SP and NPY) and SAS score (all $\mathrm{p}>0.05$ ). After treatment, these items of both groups were improved (all $p<0.05$ ) and improvements in the observation group were more notable than those in the control group (all $p<0.05$ ). In terms of efficacy, the observation group got a notably higher total effective rate than the control group $(p<0.01)$. The results could be explained by the fact that neurotransmitters such as 5-HT, SS, SP and NPY in plasma participated in gastrointestinal motility and secretion function, while trimebutine maleate helped regulate them, so that patients could get gradual recovery in gastrointestinal motility and secretion function and improvement in rectal manometry indices and their anxiety was naturally alleviated. In terms of improvement of various indices, the observation group was superior to the control group, because HBO therapy could further alleviate the anxiety of patients and improves their sleep quality, thus further improving various indexes of the body. Its mechanism may be as follows ${ }^{[16]}$ : The increase in blood flow of vertebral artery under HBO (under 2ATA oxygen pressure, the blood flow of vertebral artery can increase by $18 \%{ }^{[17]}$ ) improves the oxygen partial pressure of brain stem and reticular structure and effectively alleviates the weakening of internal inhibition process of cerebral cortex. The increase also enhances the regulation and control on subcortical autonomic nervous system function, regulates the excitatory and inhibitory function of the cerebral cortex, reduces intestinal mucosal exudation and mucus secretion and finally promotes the recovery of patients with the disease; Under HBO, the partial pressure of oxygen of the patient increases and 2ATA oxygen pressure enhances the ability of oxygen dissolution in blood by 13 times $^{[17]}$. $\mathrm{HBO}$ reduces the aggregation of platelets, inhibits the massive release of platelets 5-HT and accelerates the consumption of it. In this way, it prevents vasospasm and improves cerebral microcirculation. Finally, it allows the brain tissue to get enough oxygen and thereby increases the metabolic rate of brain tissue and promotes the recovery of dysfunctional cerebral cortex; HBO increases the diffusion of blood oxygen in the whole body and thus benefits oxygen supply and oxygen reserve of tissue cells and various metabolic abilities in the body. In this way, it corrects the disorder of autonomic nerve function, increases vasopressin secretion and microvascular permeability and promotes intestinal peristalsis and metabolic recovery; as a suggestive treatment, HBO enhances the patients' confidence in rehabilitation, reduces their anxiety, improves their dietary status and sleep quality, and thus promotes the recovery of gastrointestinal function and mental improvement. In addition, without toxic side effects, HBO reduces the dependence of patients on drugs for gastrointestinal diseases and psychotropic drugs, and greatly reduces the side effects and medication risks of patients. To sum up, for patients with IBS, trimebutine maleate combined with HBO therapy not only helps restore their gastrointestinal function, but also significantly ameliorates their negative psychology and sleep quality. In addition, it has no other toxic and side effects. Therefore, the combination treatment is worthy of clinical application. 
TABLE 1: COMPARISON OF GENERAL STATISTICAL DATA BETWEEN THE TWO GROUPS

\begin{tabular}{|c|c|c|c|c|c|c|c|}
\hline & & & & Gender & $\begin{array}{l}\text { Type of } \\
\text { disease }\end{array}$ & Education & $\begin{array}{c}\text { Type of } \\
\text { occupation }\end{array}$ \\
\hline & $\mathrm{n}$ & Age & $\begin{array}{l}\text { Course of } \\
\text { disease/m }\end{array}$ & Male/Female & $\begin{array}{l}\text { Diarrhoea/ } \\
\text { Constipation/ } \\
\text { Mix }\end{array}$ & $\begin{array}{l}\text { College } \\
\text { degree and } \\
\text { above/middle } \\
\text { School or } \\
\text { High School/ } \\
\text { Elementary } \\
\text { school and } \\
\text { below }\end{array}$ & $\begin{array}{c}\text { Migrant } \\
\text { workers/ } \\
\text { Management } \\
\text { staff/ } \\
\text { Company } \\
\text { employee }\end{array}$ \\
\hline Observation & 90 & $44.6 \pm 6.5$ & $14.53 \pm 8.17$ & $38 / 52$ & $30 / 22 / 38$ & $35 / 36 / 19$ & $5 / 46 / 39$ \\
\hline Control & 90 & $44.8 \pm 6.8$ & $14.32 \pm 6.89$ & $44 / 46$ & $33 / 25 / 32$ & $34 / 39 / 17$ & $6 / 44 / 40$ \\
\hline$t / \chi^{2}$ & & -1.918 & 0.912 & 0.806 & 0.849 & 0.246 & 0.154 \\
\hline $\mathrm{p}$ & & 0.058 & 0.364 & 0.369 & 0.654 & 0.884 & 0.926 \\
\hline
\end{tabular}

TABLE 2: COMPARISON OF EFFICACY BETWEEN TWO GROUPS AFTER TREATMENT [CASE (\%)]

\begin{tabular}{lccccc}
\hline & $\mathrm{n}$ & Cure & Markedly effective & Effective & Total effective rate \\
\hline Observation & 90 & $6(6.7)$ & $37(41.1)$ & $40(44.4)$ & 92.2 \\
Control & 90 & $2(2.2)$ & $23(26.6)$ & $39(38.1)$ & 66.9 \\
$\mathrm{t} / \chi^{2}$ & & & & 13.6 \\
$\mathrm{p}$ & & & & $<0.01$ \\
\hline
\end{tabular}

TABLE 3: COMPARISON OF 5-HT, SS, SP AND NPY BETWEEN THE TWO GROUPS BEFORE AND AFTER TREATMENT ( $\mathrm{X} \pm \mathrm{s})$

\begin{tabular}{|c|c|c|c|c|c|}
\hline & \multirow{2}{*}{$\mathrm{n}$} & \multicolumn{2}{|c|}{$5-\mathrm{HT} /(\mathrm{pg} / \mathrm{l})$} & \multicolumn{2}{|c|}{$\mathrm{SS} /(\mathrm{pg} / \mathrm{ml})$} \\
\hline & & Before treatment & After treatment* & Before treatment & After treatment* \\
\hline Observation & 90 & $109.71 \pm 23.53$ & $57.54 \pm 16.12$ & $17.46 \pm 5.41$ & $12.25 \pm 5.37$ \\
\hline Control & 90 & $108.94 \pm 24.78$ & $81.56 \pm 13.18$ & $17.53 \pm 4.89$ & $14.49 \pm 5.05$ \\
\hline $\mathrm{t}$ & & 0.468 & 23.756 & 0.498 & 4.471 \\
\hline \multirow[t]{3}{*}{$p$} & & 0.745 & $<0.01$ & 0.589 & $<0.01$ \\
\hline & $n$ & \multicolumn{2}{|c|}{$\mathrm{SP} /(\mathrm{ng} / \mathrm{l})$} & \multicolumn{2}{|c|}{$\mathrm{NPY} /(\mathrm{pg} / \mathrm{l})$} \\
\hline & $\mathrm{n}$ & Before treatment & After treatment ${ }^{*}$ & Before treatment & After treatment* \\
\hline Observation & 90 & $74.45 \pm 7.18$ & $41.37 \pm 7.16$ & $66.98 \pm 6.48$ & $90.13 \pm 14.31$ \\
\hline Control & 90 & $74.13 \pm 6.99$ & $64.89 \pm 8.23$ & $67.13 \pm 6.47$ & $76.57 \pm 10.52$ \\
\hline $\mathrm{t}$ & & 0.789 & 15.789 & 0.654 & 18.457 \\
\hline $\mathrm{p}$ & & 0.478 & $<0.01$ & 0.758 & $<0.01$ \\
\hline
\end{tabular}

Note: $p<0.01$ vs. the situation before treatment

TABLE 4: COMPARISON OF RECTAL MANOMETRY INDICES BETWEEN THE TWO GROUPS (X士S)

\begin{tabular}{lccccc}
\hline & \multirow{2}{*}{$\mathrm{n}$} & \multicolumn{2}{c}{ Rectal sensation threshold/ml } & \multicolumn{2}{c}{ Rectal defecation threshold/ml } \\
\cline { 3 - 5 } & & Before treatment & After treatment* & Before treatment & After treatment* \\
\hline Observation & 90 & $29.13 \pm 5.51$ & $36.22 \pm 5.46$ & $78.46 \pm 7.16$ & $97.53 \pm 10.13$ \\
Control & 90 & $29.16 \pm 5.42$ & $31.45 \pm 5.98$ & $78.53 \pm 7.29$ & $87.37 \pm 9.21$ \\
$\mathrm{t}$ & & 0.436 & 3.417 & 0.365 & 12.751 \\
$\mathrm{p}$ & & 0.716 & $<0.01$ & 0.658 & $<0.01$ \\
\hline
\end{tabular}




\begin{tabular}{|c|c|c|c|c|c|}
\hline & \multirow{2}{*}{$\mathrm{n}$} & \multicolumn{2}{|c|}{ Rectal pain threshold } & \multicolumn{2}{|c|}{ Rectal motor index/mmHg \% } \\
\hline & & Before treatment & After treatment* & Before treatment & After treatment* \\
\hline Observation & 90 & $138.93 \pm 16.69$ & $161.87 \pm 19.76$ & $61.13 \pm 21.42$ & $29.73 \pm 14.78$ \\
\hline Control & 90 & $139.13 \pm 16.09$ & $153.69 \pm 18.63$ & $60.93 \pm 22.53$ & $42.54 \pm 19.53$ \\
\hline $\mathrm{t}$ & & 0.789 & 15.789 & 0.984 & 19.453 \\
\hline $\mathrm{p}$ & & 0.457 & $<0.01$ & 0.631 & $<0.01$ \\
\hline
\end{tabular}

Note: $p<0.01$ vs. the situation before treatment

TABLE 5: COMPARISON OF SAS SCORE BETWEEN THE TWO GROUPS BEFORE AND AFTER TREATMENT (X士s, POINTS)

\begin{tabular}{|c|c|c|c|c|c|}
\hline & $\mathbf{n}$ & Before treatment & $\begin{array}{c}\text { After } 4 \mathrm{w} \text { of } \\
\text { treatment }\end{array}$ & $\mathrm{t}$ & $\mathrm{p}$ \\
\hline Observation & 90 & $59.14 \pm 6.00$ & $48.32 \pm 4.841$ & 10.247 & $<0.01$ \\
\hline Control & 90 & $58.97 \pm 6.78$ & $54.63 \pm 4.96$ & 7.505 & $<0.01$ \\
\hline $\mathrm{t}$ & & 0.586 & 14.783 & & \\
\hline$p$ & & 0.586 & $<0.01$ & & \\
\hline
\end{tabular}

\section{Acknowledgements:}

Xinli Feng and Yamei Wang have contributed equally to this work.

\section{Conflicts of interest}

The authors report no conflicts of interest.

\section{REFERENCES}

1. Guo J, Ma L, Xu X. Discussion on the treatment of diarrheatype irritable bowel syndrome with wind medicine. Chin J Basic Med Tradit Chin Med 2019;25(2):166-7.

2. Wenjuan F, Xuan Z, Xiucai F. The role of immune factors in the pathogenesis of irritable bowel syndrome. Chin J Intern Med 2018;57(5):378-80.

3. Guozhong M, Xiaojiang J, Faguo L, Zhengquan F, Yazhen L. Comparison and analysis of the level of awakening in patients with anxiety and primary insomnia. Chin Med Her 2012;9(29):29-31.

4. Donghui W, Yingzhen X, Haiqing Y. Preliminary clinical observation of insomnia with abnormal intestinal function. Chin J Basic Med Tradit Chin Med 2017;23(8):1165-1169.

5. Gupta S, Masand P, Kaplan D, Bhandary A, Hendricks S. The relationship between schizophrenia and irritable bowel syndrome (IBS). Schizophr Res 1997;23(3):265-8.

6. Jie Z, Yanjing G, Xiaoming F. Research progress in the diagnosis and drug treatment of irritable bowel syndrome. World Clinic Drug 2019;40:302-9.

7. Nie Yuqiang, Li Yuyuan. Classification and diagnostic criteria of Roman II functional gastrointestinal diseases. Int J Internal Med 2001;28(4):147-50.

8. Lin A, Shengsheng Z, Yang X. The effect of strengthening the spleen and soothing the liver on the quality of life of patients with diarrheal irritable bowel syndrome. Beijing Tradit Chin Med 2012;31(6):437-40.

9. Jun M, Xing S, Fen W. Correlation analysis of free fatty acids with anxiety and depression in elderly patients with chronic heart failure. Chin J Geriatr 2019;38(7):733-6.
10. Li L, Yan L, Xiongying B. A randomized controlled trial of acupuncture at Feiteng eight legal points for improving symptoms and anxiety of spleen and stomach weakness and diarrhea-type irritable bowel syndrome. J Acupunct Moxibustion 2018;34(6):12-5.

11. Zhang T, Fang J, Pan F. The role of PKA/PKC-mediated TRPV1 sensitization in irritable bowel syndrome. J Pract Med 2017;33(2):319-21.

12. Yunyun Z, Zhiping J. The efficacy of live Clostridium butyricum tablets combined with trimebutine maleate and oryzanol on patients with IBS. Chin J Microecol 2019;4:43941.

13. Fu Gaoming. The difference between atmospheric oxygen and hyperbaric oxygen. Chin Med Equip 2005(11):35.

14. Zhang Y, Zheng H, Yang Y, Wang Z. The effect of hyperbaric oxygen on the lateral limb circulation of middle cerebral artery infarction. Chin Med Equip 2014;11(S2):30-31.

15. Hongping $X$. Hyperbaric oxygen treatment of irritable bowel syndrome. Chin J Clin Rehabilit 2003;9:1455.

16. Dongmei L, Yunyan S, Enli L. Comparison and analysis of combined hyperbaric oxygen treatment for sleep quality and other unhealthy conditions of patients with insomnia. Chin Med Assoc Hyperbaric Med 2007:185-7.

17. Hayakawa T. Hyperbaric oxygen treatment in neurology and neurosurgery. J Life Sci 1974;4(1):1-25.

This is an open access article distributed under the terms of the Creative Commons Attribution-NonCommercial-ShareAlike 3.0 License, which allows others to remix, tweak, and build upon the work non-commercially, as long as the author is credited and the new creations are licensed under the identical terms

This article was originally published in a special issue, "Diagnostic and Therapeutic Advances in Biomedical Research and Pharmaceutical Sciences"

Indian J Pharm Sci 2021:83(5) spl issue "148-152" 